\title{
New phase in Chern-Simons theory on lens space
}

\author{
Kushal Chakraborty $\odot^{*}$ and Suvankar Dutta ${ }^{\dagger}$ \\ Indian Institute of Science Education \& Research Bhopal, Bhopal Bypass, Bhopal 462066, India
}

(Received 5 March 2021; accepted 6 July 2021; published 21 July 2021)

\begin{abstract}
We study $U(N)_{k}$ Chern-Simons theory on lens space in Seifert framing and write down the partition function as a unitary matrix model. In the large $k$ and large $N$ limit the eigenvalue density satisfies an upper cap $\frac{1}{2 \pi \lambda}$ where $\lambda=N /(k+N)=$ fixed. The eigenvalue density of the standard gapped phase saturates the upper cap at a critical value of $\lambda$ and ceases to exist beyond that. We find a new phase (cap-gap phase) in this theory for $\lambda$ beyond the critical value and see that the on-shell free energy for the cap-gap phase is less than that of the gapped phase.
\end{abstract}

DOI: 10.1103/PhysRevD.104.026010

\section{INTRODUCTION}

Being a topological theory the partition function (PF) of Chern-Simons (CS) theory in three dimensions is a topological invariant. At the quantum level topological invariance is also preserved but at the expense of a choice of framing $[1,2]$. The PF of CS theory with gauge group $G$, rank $k$ on Seifert manifold $M_{(g, p)}$ can be obtained by surgery from the expectation value of the Wilson loop in $S^{2} \times S^{1}$. Different choices of surgery give different framings in $M_{(g, p)}$. The PF on $M_{(g, p)}$ is given by [1]

$$
\mathcal{Z}\left(M_{(g, p)}, G, k\right)=\sum_{\mathcal{R}} \mathcal{K}_{0 \mathcal{R}}^{(p)} \sum_{\mathcal{V}} \mathcal{S}_{0, \mathcal{V}}^{1-2 g} \mathcal{S}_{\mathcal{R} \mathcal{V}}
$$

Here $\mathcal{K}^{(p)}$ is a surgery/framing dependent matrix, and $\mathcal{S}_{\mathcal{R} \mathcal{R}^{\prime}}$ is a modular transform matrix associated with highest weight representations of affine Lie algebra $g_{k}$ of $G_{k}$ under inversion of a modular parameter. The sum in (1) runs over integrable representations of $g_{k}$ and $\mathcal{R}=0$ corresponds to trivial representation. For $\mathcal{K}^{(p)}=\mathcal{S} \mathcal{T}^{-p} \mathcal{S}$ (Seifert framing), where $\mathcal{T}_{\mathcal{R R}^{\prime}}$ is the second modular transform matrix associated with the translation of the modular parameter, the $\mathrm{PF}$ is given by

$$
\mathcal{Z}^{\mathrm{SF}}\left(M_{(g, p)}, G, k\right)=\sum_{\mathcal{R}} \mathcal{S}_{0, \mathcal{R}}^{2-2 g} \mathcal{T}_{\mathcal{R} \mathcal{R}}^{-p} .
$$

Blau and Thompson [3] obtained the above PF using the method of abelianization [4]. It turns out that their

\footnotetext{
*kushal16@iiserb.ac.in

†suvankar@iiserb.ac.in
}

Published by the American Physical Society under the terms of the Creative Commons Attribution 4.0 International license. Further distribution of this work must maintain attribution to the author(s) and the published article's title, journal citation, and DOI. Funded by SCOAP. calculations render the PF in Seifert framing. Using a non-Abelian localization method one also obtains the PF in the same framing [5]. We are interested in CS theory on lens space which is a Seifert manifold with genus $g=0$ and the first Chern class $p$. For $p=1$ the Seifert manifold is $S^{3}$. On $S^{3}$, there exists a canonical framing $\mathcal{K}^{(p)}=S[1,3]$ in which the $\mathrm{PF}$ is given by $\mathcal{Z}^{\mathrm{Can}}\left(S^{3}, G, k\right)=\mathcal{S}_{00}$. Using the properties of $\mathcal{S}$ and $\mathcal{T}$ matrices $\left[\mathcal{S}^{2}=(\mathcal{S T})^{3}=\mathbb{I}\right]$ one can show that PF on $S^{3}$ in canonical and Seifert framings are related by $\mathcal{Z}^{\mathrm{SF}}\left(S^{3}, G, k\right)=\mathcal{T}_{00}^{2} \mathcal{S}_{00}$. In this paper we focus on the affine gauge group $G=U(N)_{k}$. In the large $k$ and large $N$ limit keeping

$$
\lambda=\frac{N}{k+N}
$$

fixed, one can compute the PF in Seifert framing (2) under the saddle point approximation and find the dominant representation for $0 \leq \lambda \leq 1$ [6-9]. But the dominant representations fail to be integrable for all values of $\lambda$. It was first pointed out in [10]. In this paper we address this issue in detail. Using the fact that the sum in (2) runs over integrable representations, we write the $\mathrm{PF}$ (for $g=0$ ) as a unitary matrix model. In the large $k, N$ limit the eigenvalue density is constrained to have a maximum value $\frac{1}{2 \pi \lambda}$. We derive the saddle point equation for the eigenvalue density and find that the saddle point equation admits a gapped solution for $\lambda>0$. As a consistency check, we compute the $\mathrm{PF}$ on $S^{3}$ on the gapped solution and see that it is equal to $\mathcal{T}_{00}^{2} \mathcal{S}_{00}$ for all values of $0 \leq \lambda \leq 1$. Therefore the gapped phase is equivalent to the dominant representation obtained in [6-9]. However, the eigenvalue density of the gapped phase saturates the upper bound at $\lambda=1 / \pi \log \cosh \pi / p \equiv$ $\lambda^{*}$ and ceases to exists beyond $\lambda^{*}[10]$. In this paper we discover that there exists another phase (we call this phase cap-gap phase) for $\lambda>\lambda^{*}$ for $p \geq 1$. We compute the free 
energy of the cap-gap phase and see that it is less than that of the gapped phase for $\lambda>\lambda^{*}$. Therefore our calculation shows that the PF of CS theory on lens space (2) admits a phase transition at $\lambda=\lambda^{*}$ in the large $k, N$ limit when we consider the integrability condition properly. The advantage of converting the PF to a unitary matrix model is that the dominant representations for both the gapped and capgap phases are integrable by construction.

CS on lens space also enjoys the level-rank duality [11]. We find the Young diagram (YD) distribution for a large $k$, $N$ phase and its dual and show that they are related by transposition followed by a shift. We also check that the PFs of dual theories are the same in the large $k, N$ limit. The dual of a gapped phase has an upper cap in the eigenvalue distribution. On the other hand, the cap-gap phase is dual to itself. This is similar to the matter CS theories on $S^{2} \times S^{1}$ studied by $[12,13]$.

\section{II. $U(N)$ CHERN-SIMONS THEORY ON SEIFERT MANIFOLD}

The affine Lie algebra $u(N)_{k}$ is the quotient of $s u(N)_{k} \times$ $u(1)_{N(k+N)}$ by $\mathbb{Z}_{N}$. Hence $u(N)$ representation can be written in terms of $s u(N)$ representations and eigenvalues of a $u(1)$ generator: $\mathcal{R}=(R, Q)$. We use the notation $R$ for $s u(N)$ representations and $Q$ is the eigenvalue of the $u(1)$ generator, given by $Q=r(R) \bmod N$, where $r(R)$ is the number of boxes in $R$. Trivial representation $\mathcal{R}=0$ corresponds to $R=0$ and $Q=0$. The modular transform matrix $\mathcal{S}_{\mathcal{R R}^{\prime}}$ for $u(N)_{k}$ can be written in terms of representations of $s u(N)$ and the $u(1)$ charges $[11,14,15]$

$$
\mathcal{S}_{\mathcal{R R}^{\prime}}=\frac{(-i)^{\frac{N(N-1)}{2}}}{(k+N)^{\frac{N}{2}}} e^{-\frac{2 \pi i Q Q^{\prime}}{N(N+k)}} \operatorname{det} M\left(R, R^{\prime}\right)
$$

where, $M\left(R, R^{\prime}\right)$ is an $N \times N$ matrix with elements,

$$
\begin{gathered}
M_{i j}\left(R, R^{\prime}\right)=\exp \left[\frac{2 \pi i}{k+N} \phi_{i}(R) \phi_{j}\left(R^{\prime}\right)\right], \\
\phi_{i}(R)=l_{i}-\frac{r(R)}{N}-i-\frac{1}{2}(N+1)
\end{gathered}
$$

and $l_{i}$ 's are the number of boxes in $i$ th row in $R$. The other modular transformation matrix $\mathcal{T}_{\mathcal{R R}^{\prime}}$ is given by

$$
\mathcal{T}_{\mathcal{R R}^{\prime}}=e^{2 \pi i\left(h_{R}-\frac{c}{24}\right)} \delta_{\mathcal{R R}^{\prime}}, \quad h_{R}=\frac{1 C_{2}(\mathcal{R})}{2} \frac{k+N}{k+N}, \quad c=\frac{N(N k+1)}{k+N}
$$

where $\mathcal{C}_{2}(\mathcal{R})$ is the quadratic Casimir of $u(N)_{k}$. Since $Q=$ $r(R)+N s$ for $s \in \mathbb{Z}, u(N)$ representations $\mathcal{R}$ can be characterized by extended YDs by redefining the number of boxes in the $i$ th row $\bar{l}_{i}=l_{i}+s$ for $1 \leq i \leq N-1$ and $\bar{l}_{N}=s$. Now $\bar{l}_{i}$ s can be negative and the corresponding
YDs will have antiboxes [16]. In terms of these extended YDs the quadratic Casimir $C_{2}(\mathcal{R})$ is given by

$$
\mathcal{C}_{2}(\mathcal{R})=\sum_{i=1}^{N} \bar{l}_{i}\left(\bar{l}_{i}-2 i+N+1\right) .
$$

A representation $\mathcal{R}$ of $u(N)_{k}$ is an integrable representation if $0 \leq \bar{l}_{N} \leq \cdots \leq \bar{l}_{1} \leq k[11]$.

\section{CHERN-SIMONS THEORY AS UNITARY MATRIX MODEL}

For an integrable representation $\mathcal{R}$ the hook numbers $h_{i}=\bar{l}_{i}+N-i$ satisfy $0<h_{N}<\cdots<h_{1} \leq k+N$. Introducing new variables

$$
\theta_{i}=\frac{2 \pi}{N+K}\left(h_{i}-\frac{N-1}{2}\right)
$$

we write the CS partition function (2) for $g=0$ in terms of $\theta_{i} \mathrm{~s}\left[\mathcal{Z}^{\mathrm{SF}}\left(M_{(0, p)}, U(N), k\right) \rightarrow \mathcal{Z}_{N, k}^{p}\right]$

$$
\begin{aligned}
\mathcal{Z}_{N, k}^{p}= & \frac{1}{(N+k)^{N}} \sum_{\left\{\theta_{i}\right\}} \exp \left[\frac{1}{2} \sum_{i \neq j}^{N} \log \left[4 \sin ^{2}\left(\frac{\theta_{i}-\theta_{j}}{2}\right)\right]\right. \\
& \left.-i p\left(\frac{N+k}{\pi} \sum_{i=1}^{N}\left(\frac{\theta_{i}^{2}}{4}-\frac{\pi^{2}}{12}\right)+\frac{\pi N K}{12}\right)\right] .
\end{aligned}
$$

The effective action (argument of the exponential) is symmetric in $\theta_{i} \rightarrow-\theta_{i}$. Since the distribution of $\theta_{i}$ s has a maximum range $2 \pi$ [from Eq. (9)] any classical configuration will satisfy $-\pi \leq \theta_{i} \leq \pi$. The potential is neither real nor periodic. However, in order to write a unitary matrix model for CS theory we demand that $\theta_{i}$ s are periodic with periodicity $2 \pi$ which essentially means that we impose periodicity in hook numbers $\bar{h}_{i} \mathrm{~s}: \bar{h}_{i} \sim \bar{h}_{i}+k+N$. To make the potential real we analytically continue $p \rightarrow-i p$. This allows us to write the above partition function as a unitary matrix model with potential $\sim \sum_{n>0} \frac{(-1)^{2}}{2 n^{2}}\left(\operatorname{Tr} U^{n}+\operatorname{Tr} U^{\dagger n}\right)$ [17]. Later we see that in the large $k, N$ limit the on-shell partition function on $S^{3}$ matches with $\mathcal{S}_{00}$ (up to a phase) when we carefully replace $p \rightarrow i p$.

In the continuum limit we define an eigenvalue density $\rho(\theta)=\frac{1}{N} \sum_{i=1}^{N} \delta\left(\theta-\theta_{i}\right)$. The partition function is given by

$$
\begin{aligned}
\mathcal{Z}_{N, k}^{p}= & \int[d \theta] e^{-(N+K)^{2} S_{\mathrm{eff}}[\rho]}, \quad \text { where } \\
S_{\mathrm{eff}}[\rho]= & \frac{p \lambda}{\pi} \int \rho(\theta)\left(\frac{\theta^{2}}{4}-\frac{\pi^{2}}{12}\right) d \theta+\frac{\pi p \lambda(1-\lambda)}{12} \\
& -\frac{\lambda^{2}}{2} \int f \rho(\theta) \rho\left(\theta^{\prime}\right) \log \left[4 \sin ^{2}\left(\frac{\theta-\theta^{\prime}}{2}\right)\right] d \theta d \theta^{\prime} .
\end{aligned}
$$


The saddle point equation for $\rho(\theta)$, obtained from this effective action is given by

$$
f \rho\left(\theta^{\prime}\right) \cot \left(\frac{\theta-\theta^{\prime}}{2}\right) d \theta^{\prime}=\frac{p}{2 \pi \lambda} \theta .
$$

From the definition of $\theta_{i} \mathrm{~s}(9)$ we see that the minimum separation between $\theta_{i}$ and $\theta_{i+1}$ is $2 \pi /(N+k)$. This implies that in the large $k, N$ limit the eigenvalue density $\rho(\theta)$ satisfies an upper bound

$$
\rho(\theta) \leq \frac{1}{2 \pi \lambda} .
$$

Therefore we have to solve the saddle point equation (12) for $\rho(\theta)$ in the presence of this constraint. Note that the YD distribution can be obtained from the eigenvalue distribution: $u(h)=2 \pi \lambda \rho(\theta)$. Hence, $\rho(\theta)$ having an upper cap $1 / 2 \pi \lambda$ means $u(h) \leq 1$. Before we discuss the large $k, N$ phases of this theory we take a pause to study the eigenvalue density of the level-rank dual theory and its connection to $\rho(\theta)$.

\section{EVEL-RANK DUALITY}

$N \leftrightarrow k$ duality in $U(N)$ CS theory implies that the dominant YDs in two theories dual to each other are related by a transposition followed by a shift. In order to prove this statement we write down the partition function of $U(N)_{k}$ CS theory in terms of number of boxes in different columns in a YD. A YD corresponding to an integrable representation of $u(N)_{k}$ can be characterized by $\bar{v}_{\mu}$-the number of boxes in the $\mu$ th column of a YD $\mathcal{R}$ where $1 \leq \mu \leq k$ and $\bar{v}_{1} \leq N .\left\{\bar{v}_{\mu}\right\}$ is the set of box numbers in different rows of $\tilde{\mathcal{R}}$, where $\tilde{\mathcal{R}}$ is the transpose of $\mathcal{R}$. The quadratic Casimir $C_{2}(\mathcal{R})$ can be written in terms of $\bar{v}_{\mu}$. Also the $\mathcal{S}$ modular transform matrix (4) is invariant under transposition [11]. We introduce new variables

$\phi_{\mu}=\frac{2 \pi}{k+N}\left(w_{\mu}-\frac{k+N-1}{2}\right), \quad w_{\mu}=\bar{v}_{\mu}+k-\mu$.

Since $0 \leq \bar{v}_{\mu} \leq N, \phi_{\mu}$ s are distributed in a range of $2 \pi$. The partition function (2) can be written in terms of $\phi_{\mu} \mathrm{s}$ and it turns out that the effective action is symmetric under $\phi_{\mu} \rightarrow 2 \pi-\phi_{\mu}$. Hence for any classical solution $\phi_{\mu}$ s are distributed symmetrically about $\phi=\pi$ from 0 to $2 \pi$. In the continuum limit we define a distribution function for $\phi_{\mu} \mathrm{s}$

$$
\tilde{\rho}(\phi)=\frac{1}{k} \sum_{\mu=1}^{k} \delta\left(\phi-\phi_{\mu}\right)
$$

and the partition function is given by

$$
\mathcal{Z}_{N, k}^{p}=\int[d \phi] e^{-(N+k)^{2} \tilde{S}_{\text {eff }}[\tilde{\rho}]}
$$

where

$$
\begin{aligned}
\tilde{S}_{\mathrm{eff}}[\tilde{\rho}]= & \frac{p \tilde{\lambda}}{\pi} \int \tilde{\rho}(\phi)\left(\frac{\pi^{2}}{12}-\frac{(\phi-\pi)^{2}}{4}\right) d \phi+\frac{p \pi \lambda \tilde{\lambda}}{12} \\
& -\frac{\tilde{\lambda}^{2}}{2} \int f \tilde{\rho}(\phi) \tilde{\rho}\left(\phi^{\prime}\right) \log \left(4 \sin ^{2}\left(\frac{\phi-\phi^{\prime}}{2}\right)\right) d \phi d \phi^{\prime}
\end{aligned}
$$

and $\tilde{\lambda}=1-\lambda$. The saddle point equation for $\tilde{\rho}(\phi)$ is given by

$$
f--_{0}^{2 \pi} \tilde{\rho}\left(\phi^{\prime}\right) \cot \left(\frac{\phi-\phi^{\prime}}{2}\right) d \phi^{\prime}=\frac{p}{2 \pi \tilde{\lambda}}(\pi-\phi) .
$$

Comparing (12) and (18) we find

$$
\tilde{\rho}(\phi)=\frac{1}{2 \pi \tilde{\lambda}}-\frac{\lambda}{\tilde{\lambda}} \rho(\phi+\pi) .
$$

This relation establishes the fact that under $N \leftrightarrow k$ duality the dominant YDs in $U(N)_{k}$ and $U(k)_{N}$ CS theories are related by a transposition with a shift $\frac{N+k}{2}$. Using (19) we also see that $S[\rho]=\tilde{S}[\tilde{\rho}]$. The relation (19) is similar to what was found in $[10,12]$ in the context of matter CS theory on $S^{2} \times S^{1}$.

\section{LARGE $N$ PHASES}

The unitary matrix model (11) was studied in $[10,18]$. It was observed that the system has a gapped phase in the large $k, N$ limit and the eigenvalue distribution is given by

$$
\rho(\theta)=\frac{p}{2 \pi^{2} \lambda} \tanh ^{-1}\left[\sqrt{1-\frac{e^{-\frac{2 \pi \lambda}{p}}}{\cos ^{2} \frac{\theta}{2}}}\right] .
$$

Since $\rho(\theta) \geq 0$, this implies eigenvalues are distributed over the range

$$
-2 \cos ^{-1} e^{-\frac{\pi \lambda}{p}}<\theta<2 \cos ^{-1} e^{-\frac{\pi \lambda}{p}} .
$$

See Fig. 1 for the eigenvalue distribution. We calculate the PF (10) on $S^{3}$ (i.e. for $p=1$ ) on this solution (20) and check that after suitable analytic continuation $p \rightarrow i p$ (before setting $p=1$ ) the PF exactly matches with $\mathcal{T}_{00}^{2} \mathcal{S}_{00}$ for all values of $0 \leq \lambda \leq 1$. Hence this phase is equivalent to the dominant phase obtained in [6-9]. However, due to the constraint (13) on $\rho(\theta)$ the eigenvalue density saturates the upper bound at $\lambda=$ $1 / \pi \log \cosh (\pi / p) \equiv \lambda^{*}[10]$. Therefore the gapped phase is not valid anymore for $\lambda>\lambda^{*}$ for any $p \geq 1$. 


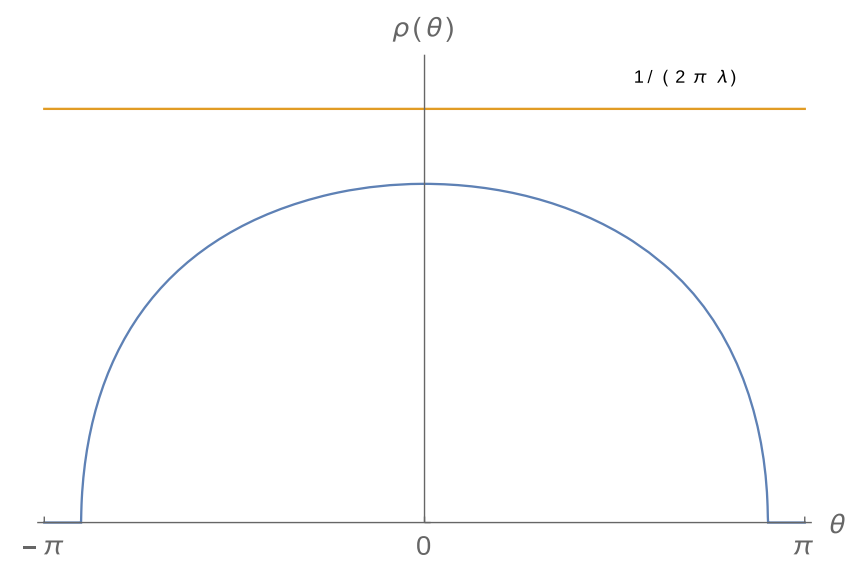

FIG. 1. $\rho(\theta)$ for one-gap phase.

\section{CAP-GAP PHASE}

For $\lambda>\lambda^{*}$ the eigenvalue density develops a cap about $\theta=0$. To find that phase we take the following ansatz for $\rho(\theta)$ :

$\rho(\theta)=\left\{\begin{array}{l}\frac{1}{2 \pi \lambda} \quad \text { for }-\theta_{2}<\theta<\theta_{2} \\ \hat{\rho}(\theta) \text { for }-\theta_{1}<\theta<-\theta_{2} \text { and } \theta_{2}<\theta<\theta_{1} .\end{array}\right.$

Using the map $z=e^{i \theta}$, the saddle point equation for $\hat{\rho}(\theta)$ is given by

$f \hat{\rho}\left(z^{\prime}\right) \frac{z+z^{\prime}}{z-z^{\prime}} d z^{\prime}=\frac{p \log (z)}{2 \pi i \lambda}-\frac{1}{2 \pi \lambda} \int \frac{1}{z^{\prime}} \frac{z+z^{\prime}}{z-z^{\prime}} d z^{\prime}$.

Following [12] we define a resolvent function $\Phi(z)$

$\Phi(z)=\int \frac{\hat{\rho}\left(z^{\prime}\right)}{i z^{\prime}} \frac{z+z^{\prime}}{z-z^{\prime}} d z^{\prime}=h(z) H(z), \quad$ where

$h(z)=\sqrt{\left(z^{2}-2 z \cos \theta_{1}+1\right)\left(z^{2}-2 z \cos \theta_{2}+1\right)}$.

From the normalization of eigenvalue density it follows that

$$
\Phi(z \rightarrow \infty) \sim 1-\frac{1}{2 \pi \lambda} \int \frac{d \omega}{i \omega} .
$$

The resolvent $\Phi(z)$ has a branch cut in the complex $z$ plane. The eigenvalue density $\hat{\rho}(z)$ is obtained from the discontinuity of $\Phi(z)$

$$
\Phi^{+}(z)-\Phi^{-}(z)=4 \pi \hat{\rho}(z) .
$$

Following [19] the function $H(z)$ can be evaluated as

$$
H(z)=i \oint \frac{d w}{2 \pi i} \frac{\frac{p \log (w)}{2 \pi i \lambda}-\frac{1}{2 \pi \lambda} \int \frac{1}{s} \frac{w+s}{w-s} d s}{h(w)(w-z)}
$$

Plugging this expression in (24) we expand the right-hand side for large $z$ and comparing the expression with (25) we find the following two constraints:

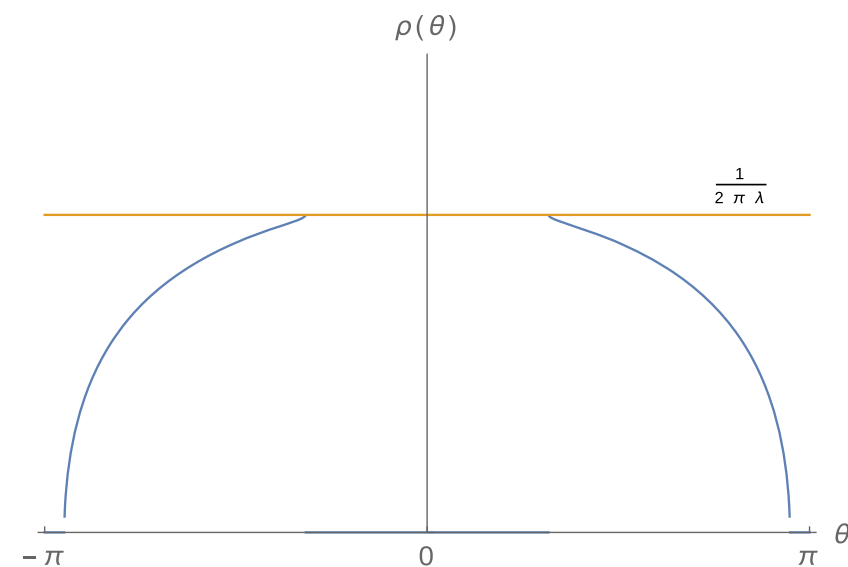

FIG. 2. $\rho(\theta)$ for cap-gap phase.

$$
\begin{aligned}
\frac{p}{2 \pi \lambda} \oint \frac{d z}{2 \pi i} \frac{\log (z)}{h(z)}+\frac{i}{\pi \lambda} \int \frac{d \omega}{h(\omega)} & =0, \\
1+\frac{p}{2 \pi \lambda} \oint \frac{d z}{2 \pi i} \frac{z \log (z)}{h(z)}+\frac{i}{\pi \lambda} \int \frac{\omega d \omega}{h(\omega)} & =0 .
\end{aligned}
$$

Using the formula given in the Appendix, we numerically solve these two equations to find the endpoints $\theta_{1}$ and $\theta_{2}$. From the discontinuity $\Phi(z)$ we compute the eigenvalue density

$$
\begin{aligned}
\hat{\rho}(\theta)= & -\frac{|\sin \phi|}{\pi^{2} \lambda} \frac{\sqrt{\left(\sin ^{2} \frac{\phi}{2}-\sin ^{2} \frac{\theta_{2}}{2}\right)\left(\sin ^{2} \frac{\theta_{1}}{2}-\sin ^{2} \frac{\phi}{2}\right)}}{\sqrt{\left(1+\cos \theta_{2}\right)\left(1-\cos \theta_{1}\right)}} \\
& \times\left[\frac{2 p\left(\cos ^{2} \frac{\theta_{1}}{2} \Pi\left(\psi, n_{1}, m_{1}\right)-\cos ^{2} \frac{\phi}{2} F\left(\psi, m_{1}\right)\right)}{(1+\cos \phi)\left(\cos \phi-\cos \theta_{1}\right)}\right. \\
& \left.-\frac{4\left(\Pi\left(n_{2}, m_{2}\right)-\sin ^{2} \frac{\phi}{2} K\left(m_{2}\right)\right)}{\sin ^{2} \phi}\right]
\end{aligned}
$$

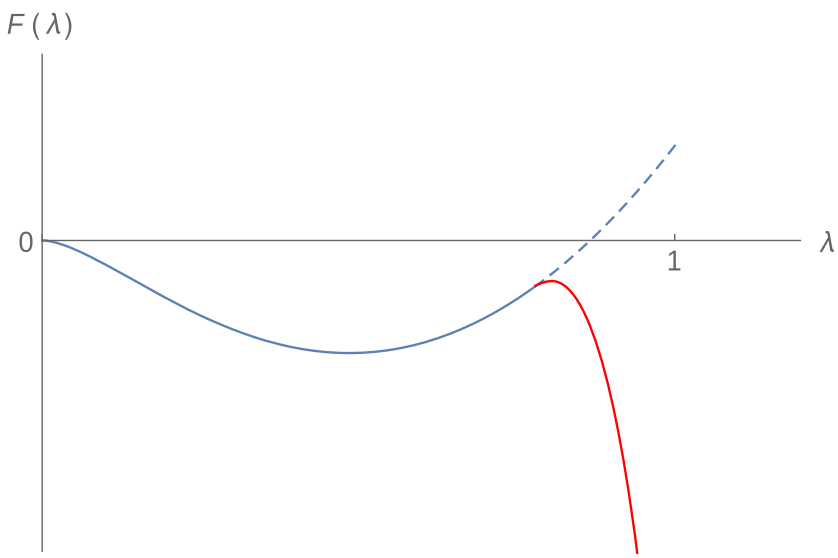

FIG. 3. Free energy of CS on $S^{3}$ as a function of $\lambda$. The solid blue line is the free energy for the gapped phase for $\lambda<\lambda^{*}$. The dashed blue line is the extension of the same beyond $\lambda^{*}$. The red line depicts the free energy for the cap-gap phase. 
where $m_{1}, m_{2}, n_{1}, n_{2}, \psi$ are given in (A3). The eigenvalue density in cap-gap phase is plotted in Fig. 2. The free energies for these two phases (for $p=1$ ) as a function of $\lambda$ are plotted in Fig. 3. From this figure we see that the capgap phase has free energy less than that of the gapped phase and hence is dominant over the gapped phase for $\lambda>\lambda^{*}$. However the on-shell $S^{3} \mathrm{PF}$ on the cap-gap phase differs from $\mathcal{T}_{00}^{2} \mathcal{S}_{00}$.

\section{DISCUSSION}

In order to obtain a real saddle point equation (12) we use an analytic continuation in $p$. However if we use an analytic continuation in $\lambda$ [10], we would also get a real saddle point equation for YD distribution $u(h)$ in the $h$ plane with a coth kernel similar to that considered in [8]. The solution of this equation renders a YD distribution which crosses the maximum value 1 for $p>2$ only. Hence one can exhibit a phase transition only for $p \geq 3$. The YD distribution is different from the one-gap eigenvalue distribution obtained in this paper. But with a proper analytic continuation of $p$ and $\lambda$ one can relate the two [10]. However, the YD distribution obtained in $[6,8,10]$ violates the integrability bound for some value of $\lambda$ between 0 and 1 . In strictly $k \rightarrow \infty$ limit the sum over $\mathcal{R}$ in (2) is unrestricted. Hence one should not expect any phase transition in the system. But when we consider the level and rank to be large but finite and take the double scaling limit properly we observe the phase transition. In the double scaling limit $\theta_{i}$ s are defined in such a way (9) that they have a range between 0 and $2 \pi$ and the dominant representations are always integrable. But this change of variables imposes a cap on the eigenvalue distribution which triggers a phase transition in the theory.

The 't Hooft expansion of the PF of $S U(N)$ CS theory on $S^{3}$ is proposed to be dual to topological closed string theory on the $S^{2}$ blow up of the conifold geometry [20] for arbitrary $\lambda$ and all orders of $1 / N$. In canonical framing the CS PF is equal to $\mathcal{S}_{00}$ and an exact function of $\lambda$ which matches with the string theory side. In Seifert framing, we observe that the PF of CS theory in the gapped phase is equal to that in the string theory side. But the PF in cap-gap phase differs from $\mathcal{S}_{00}$ for $\lambda>\lambda^{*}$. Dependence of phase on the choice of framing is a bit puzzling here. The question is why a new phase pops up in the theory when we take the double scaling limit. The saddle equation (12) also admits multicut solutions, which were related to some nonperturbative $D$-instantons [21]. These multi-cut solutions are different than the cap-gap phase (29) studied in this paper. It would be interesting to understand the meaning of this new phase in the string theory side as well.

We explicitly check the level-rank duality in CS theory on $S^{3}$. The theory admits three types of phases. For $\lambda<\lambda^{*}$ one has a gapped phase and a capped phase. These two phases are level-rank dual to each other. For $\lambda>\lambda^{*}$ the theory admits a cap-gap phase which is level rank dual to itself. There is a third order phase transition at $\lambda^{*}$. The phase structure is similar to that of CS-matter theory on $S^{2} \times S^{1}$ $[10,12]$ except that here we do not have any gapless phase.

The partition function of $q$-deformed $U(N)$ Yang-Mills on a generic Riemann surface with zero $\theta$ term is equal to the PF of CS theory on $M_{(g, p)}$ up to a phase factor for $q=e^{\frac{2 \pi i}{N+k}}$ and $k, p \in \mathbb{Z}$ [11]. Thus our analysis shows that the $q$-deformed Yang-Mills undergoes a phase transition even for $p=1$ unlike [8].

\section{ACKNOWLEDGMENTS}

We thank Arghya Chattopadhyay and Neetu for working on this problem at the initial stage. We are grateful to Rajesh Gopakumar and Dileep Jatkar for reading our manuscript and giving their valuable comments. The work of S.D. is supported by the MATRICS Grant No. MTR/ 2019/000390, the Department of Science and Technology, Government of India.

\section{APPENDIX: USEFUL FORMULA}

We use the following useful results in our calculations:

$$
\begin{aligned}
\oint \frac{d z}{2 \pi i} \frac{\log (z)}{h(z)} & =\frac{2 F\left(\psi, m_{1}\right)}{\sqrt{\left(1+\cos \theta_{2}\right)\left(1-\cos \theta_{1}\right)}}, \\
\oint \frac{d z}{2 \pi i} \frac{z \log (z)}{h(z)} & =\frac{2 \cos \theta_{1} F\left(\psi, m_{1}\right)}{\sqrt{\left(1+\cos \theta_{2}\right)\left(1-\cos \theta_{1}\right)}}+\frac{2 \beta v_{4}^{\prime}(\beta)}{v_{4}(\beta)}+\frac{1}{2} \log \left(\frac{\left(1-\cos \theta_{1}\right)\left(1+\cos \theta_{2}\right)}{4 K^{2}\left(m_{1}\right)}\right)-\log \left[\frac{v_{1}(2 \beta)}{v_{1}^{\prime}(0)}\right]
\end{aligned}
$$

where,

$$
\begin{gathered}
\beta=\frac{F\left(\psi, m_{1}\right)}{2 K\left(m_{1}\right)}, \quad q=e^{-\pi \frac{K^{\prime}\left(m_{1}\right)}{K\left(m_{1}\right)}}, \quad K^{\prime}\left(m_{1}\right)=K\left(\sqrt{1-m_{1}^{2}}\right) \\
\int_{e^{-i \theta_{2}}}^{e^{i \theta_{2}}} \frac{d \omega}{h(\omega)}=\frac{2 i K\left(m_{2}\right)}{\sqrt{\left(1-\cos \theta_{1}\right)\left(1+\cos \theta_{2}\right)}}, \quad \int_{e^{-i \theta_{2}}}^{e^{i \theta_{2}}} \frac{\omega d \omega}{h(\omega)}=\frac{2 i\left(2 \Pi\left(n, m_{2}\right)-K\left(m_{2}\right)\right)}{\sqrt{\left(1-\cos \theta_{1}\right)\left(1+\cos \theta_{2}\right)}},
\end{gathered}
$$




$$
\begin{aligned}
& \psi=\sin ^{-1} \sqrt{\frac{1-\cos \theta_{1}}{2}}, \quad m_{1}=\sqrt{\frac{2\left(\cos \theta_{2}-\cos \theta_{1}\right)}{\left(1+\cos \theta_{2}\right)\left(1-\cos \theta_{1}\right)}}, \quad m_{2}=\sqrt{\frac{\left(1-\cos \theta_{2}\right)\left(1+\cos \theta_{1}\right)}{\left(1-\cos \theta_{1}\right)\left(1+\cos \theta_{2}\right)}} ; \\
& n=\frac{\cos \theta_{2}-1}{1+\cos \theta_{2}} ; \quad n_{1}=\frac{2\left(\cos \phi-\cos \theta_{1}\right)}{\left(1-\cos \theta_{1}\right)(1+\cos \phi)} ; \quad n_{2}=\frac{\left(1-\cos \theta_{2}\right)(1+\cos \phi)}{\left(1+\cos \theta_{2}\right)(1-\cos \phi)} .
\end{aligned}
$$

[1] E. Witten, Commun. Math. Phys. 121, 351 (1989).

[2] M. Atiyah, Topology 29, 1 (1990).

[3] M. Blau and G. Thompson, J. High Energy Phys. 05 (2006) 003.

[4] M. Blau and G. Thompson, Nucl. Phys. B408, 345 (1993).

[5] C. Beasley and E. Witten, J. Diff. Geom. 70, 183 (2005).

[6] M. Marino, arXiv:hep-th/0410165.

[7] M. Marino, Commun. Math. Phys. 253, 25 (2005).

[8] X. Arsiwalla, R. Boels, M. Marino, and A. Sinkovics, Phys. Rev. D 73, 026005 (2006).

[9] M. Aganagic, A. Klemm, M. Marino, and C. Vafa, J. High Energy Phys. 02 (2004) 010.

[10] A. Chattopadhyay, D. Suvankar, and Neetu, Phys. Rev. D 100, 126009 (2019).

[11] S. G. Naculich and H. J. Schnitzer, J. High Energy Phys. 06 (2007) 023.
[12] S. Jain, S. Minwalla, T. Sharma, T. Takimi, S. R. Wadia, and S. Yokoyama, J. High Energy Phys. 09 (2013) 009.

[13] A. Chattopadhyay, P. Dutta, and S. Dutta, J. High Energy Phys. 05 (2018) 117.

[14] E. J. Mlawer, S. G. Naculich, H. A. Riggs, and H. J. Schnitzer, Nucl. Phys. B352, 863 (1991).

[15] P. D. Francesco, P. Mathieu, and D. Sénéchal (Springer, New York, 1997).

[16] M. Aganagic, A. Neitzke, and C. Vafa, Adv. Theor. Math. Phys. 10, 603 (2006).

[17] T. Okuda, J. High Energy Phys. 03 (2005) 047.

[18] A. Chattopadhyay, P. Dutta, and S. Dutta, J. High Energy Phys. 11 (2017) 186.

[19] A. A. Migdal, Phys. Rep. 102, 199 (1983).

[20] R. Gopakumar and C. Vafa, Adv. Theor. Math. Phys. 3, 1415 (1999).

[21] T. Morita and K. Sugiyama, Nucl. Phys. B929, 1 (2018). 